\title{
THE CHAIN RECURRENT SET FOR MAPS OF THE INTERVAL
}

\author{
LOUIS BLOCK AND JOHN E. FRANKE
}

\begin{abstract}
Let $f$ he a continuous map of a compact interval into itself. We show that if the set of periodic points of $f$ is a closed set then every chain recurrent point is perindic.
\end{abstract}

1. Introduction. The concept of chain recurrent points and the chain recurrent set was introduced by Conley $[3,4]$ in the study of flows on manifolds. This paper is concerned with the chain recurrent set of a continuous map of the interval to itself. Our main result is the following.

THEOREM. Let $f$ be a continuous map of a compact interval I into itself. If the set of periodic points of $f$ is a closed set then every chain recurrent point is periodic.

One may think of a chain recurrent point as a point that looks periodic on a computer, i.e. where error is allowed (see $\$ 2$ for the definition). Thus, the theorem asserts that (if the hypothesis is satisfied) every point that looks periodic on a computer is actually periodic.

Nitecki [8] and Ziong [10] prove that if the hypothesis of our theorem is satisfied then every nonwandering point is periodic. Previous related results and special cases may be found in [ $\mathbf{1}$ and $\mathbf{5}$ ].

Note that while the nonwandering set is always a subset of the chain recurrent set the reverse inclusion need not hold. For example. consider a map $f$ from $[0,1]$ to itself such that:

(1) $f(0)=0, f\left(\frac{1}{3}\right)=\frac{1}{3}, f\left(\frac{2}{3}\right)=1$, and $f(1)=0$.

(2) $f$ maps the interval $\left[0, \frac{1}{3}\right]$ homeomorphically onto itself.

(3) $f(x)>x$ for all $x \in\left(0, \frac{1}{3}\right)$.

(4) The restriction of $f$ to each of the intervals $\left[\frac{1}{3}, \frac{2}{3}\right]$ and $\left[\frac{2}{3}, 1\right]$ is linear.

Then each point in the interval $\left(0, \frac{1}{3}\right)$ is wandering, but chain recurrent.

Before proving the theorem, we give some definitions and obtain some basic properties of the chain recurrent set which hold for continuous maps of a compact metric space to itself.

2. Chain recurrent points and the chain recurrent set. In this section we let $f$ be a continuous map from a compact metric space $(X, d)$ into itself. Let $x, y \in X$. An $\varepsilon$-chain from $x$ to $y$ is a finite sequence of points $\left\{x_{0}, x_{1}, \ldots, x_{n}\right\}$ of $X$ with $x=x_{0}$,

Received by the the editors May 6, 1982 and, in revised form. August 24, 1982: presented to the Society. August 25. 1982 in Toronto.

1980 Mathematics Subject Classification. Primary 54H20

. 1983 American Mathematical Society 0002-9939/82/0000-0932/\$02.00 
$y=x_{n}$ and $d\left(f(x, 1), x_{1}\right)<\varepsilon$ for $i=1, \ldots, n$. We say $x$ can be chained to $y$ if for every $\varepsilon>0$ there is an $\varepsilon$-chain from $x$ to $y$, and we say $x$ is chain recurrent if $x$ can be chained to itself. The set of all chain recurrent points is called the chain recurrent set and denoted by $R(f)$.

For any nonnegative integer $n$ we define $f^{n}$. inductively by letting $f^{\prime \prime}$ be the identity map and $f^{n}=f \circ f^{n-1}$. A point $x \in X$ is called a periodic point of $f$ if $f^{\prime \prime}(x)=x$ for some positive integer $n$, and the smallest such $n$ is called the period of $x$.

The following property of the chain recurrent set (which may be easily verified) is false for the nonwandering set [6].

LEMMA 1. $R(f)=R\left(f^{n}\right)$.

Lemma 2. If $x \in R(f)$ then $f^{h}(x)$ can be chained to $x$ for every positive integer $k$.

Proof. Since the conclusion follows immediately if $x$ is a periodic point of $f$. we may assume that $x$ is not periodic. Let $\delta_{1}$ be the distance from $x$ to the set $\left\{f(x), f^{2}(x) \ldots, f^{h+1}(x)\right\}$ and let $\varepsilon>0$. By the uniform continuity of $f, f^{2}, \ldots f^{h+1}$. there is a $\delta>0$ such that if $y, z \in X$ and $d(y, z)<\delta$ then $d\left(f^{\prime}(y), f^{\prime}(z)\right)<$ $\min \left\{\varepsilon /(k+1), \delta_{1} /(k+1)\right\}$ for $i=0,1, \ldots, k+1$. Let $\left\{x_{0}, x_{1} \ldots, x_{n}\right\}$ be a $\delta$-chain from $x$ to $x$. Note that for $i=1, \ldots k+1$,

$$
\begin{aligned}
d\left(f^{\prime}(x), x_{1}\right) \leqslant & d\left(f^{\prime}\left(x_{0}\right), f^{\prime-1}\left(x_{1}\right)\right)+d\left(f^{\prime-1}\left(x_{1}\right), f^{1-2}\left(x_{2}\right)\right) \\
& +\cdots+d\left(f\left(x_{1-1}\right), x_{1}\right) \\
= & d\left(f^{\prime-1}\left(f\left(x_{0}\right)\right), f^{\prime-1}\left(x_{1}\right)\right)+d\left(f^{\prime-2}\left(f\left(x_{1}\right)\right), f^{\prime-2}\left(x_{2}\right)\right) \\
& +\cdots+d\left(f\left(x_{1-1}\right), x_{1}\right) \\
< & \frac{\delta_{1}}{k+1}+\frac{\delta_{1}}{k+1}+\cdots+\frac{\delta_{1}}{k+1} \leqslant \delta_{1} .
\end{aligned}
$$

Since $d\left(f^{\prime}(x), x\right)>\delta_{1}$, we see that $x, \neq x$ for $i=1, \ldots, k+1$. Hence any $\delta$-chain from $x$ to $x$ must have more than $k+2$ elements. A similar argument shows that $d\left(f^{h+1}(x), x_{k+1}\right)<\varepsilon$. Hence $\left\{f^{k}(x), x_{k+1}, x_{k+2}, \ldots, x_{n}\right\}$ is an $\varepsilon$-chain from $f^{k}(x)$ to $x_{n}=x$. Q.E.D.

We say a set $Y \subset X$ is positively chain invariant if for every $y \in Y$ and $x \in X \backslash Y$, $y$ cannot be chained to $x$. The next lemma follows directly from Lemma 2 and this definition.

LemMA 3. Let $Y$ be a positively chain invariant set. If $x \notin Y$ and $f^{k}(x) \in Y$ for some positive integer $k$ then $x \notin R(f)$.

3. Proof of the Theorem. For the remainder of the paper we let $I$ denote the interval $[0,1]$ and $f$ be a continuous map of $I$ to itself.

The next lemma gives a sufficient condition for a subinterval of $I$ to be positively chain invariant.

LeMma 4. Let $[a, b]$ be a subinterval of $I=[0,1]$ with $f([a, b]) \subset[a, b]$ and $b \neq 1$. Suppose there is a neighborhood $W$ of $b$ with $f(W) \subset[a, b]$. Suppose also that either $f(a)>a$ or $a=0$. Then $[a, b]$ is positively chain invariant. 
Proof. Let $z \in[a, b]$ and $y \in I \backslash[a, b]$. We must show that $z$ cannot be chained to $y$.

Let $\varepsilon_{1}$ be a positive number smaller than the distance from $b$ to the right endpoint of $W$. If $a=0$ let $\varepsilon_{2}=\varepsilon_{1}$. If $a \neq 0$ let $\varepsilon_{2}$ be a positive number such that if $x \in\left(a-\varepsilon_{2}, a\right)$ then $f(x) \in\left(a, b+\varepsilon_{1} / 2\right)$. By choosing $\varepsilon_{1}$ and $\varepsilon_{2}$ smaller if necessary we may assume that $y \notin\left(a-\varepsilon_{2}, b+\varepsilon_{1}\right)$. Let $\varepsilon=\min \left\{\varepsilon_{2}, \varepsilon_{1} / 2\right\}$.

Suppose that $z$ can be chained to $y$. Then there is an $\varepsilon$-chain $\left\{x_{0}, x_{1} \ldots \ldots x_{n}\right\}$ from $z$ to $y$. By choice of $\varepsilon$, it follows that if $0 \leqslant k \leqslant n$ and $x_{k} \in\left(a-\varepsilon_{2}, b+\varepsilon_{1}\right)$ then $x_{k+1} \in\left(a-\varepsilon_{2}, b+\varepsilon_{1}\right)$.

Hence, each element of the chain $\left\{x_{0}, x_{1}, \ldots, x_{n}\right\}$ is in the interval $\left(a-\varepsilon_{2}, b+\varepsilon_{1}\right)$. This is a contradiction since $x_{n}=y$ and $y \notin\left(a-\varepsilon_{2}, b+\varepsilon_{1}\right)$. Q.E.D.

The following lemma is a slight modification of Lemma 6 of [1]. The proof given in [1] applies in this case.

Lemma 5. Let $W$ he an open interval in I which contains no periodic points of $f$. Suppose that if an endpoint of $W$ is not a fixed point of $f$ then it is an endpoint of $I$ and is not periodic.

Suppose also that for some $y_{0} \in W, f\left(y_{0}\right)<y_{0}$. Then for every positive integer $n$ and every $y \in W, f^{n}(y)<y$.

As noted in [8], it follows from [2] that if $f$ has a periodic point whose period is not a power of two, then the set of periodic points of $f$ is not a closed set. Thus, from [7] (or Lemma 1.6 of [9]) we obtain the following.

LEMma 6. Suppose that the set of periodic points of $f$ is a closed set, and suppose that for some $z \in I$ and some positive integer $m . f(z)<z \leqslant f^{m}(z)$. Then there is a point $s_{0} \in I$ such that $f^{\prime}(z)>s_{0}$ for $i=0,2,4, \ldots, m-2$, and $f^{\prime}(z)<s_{0}$ for $i=1$. $3.5, \ldots, m-1$.

Proof of The Theorem. Let $x \in I$ be any point which is not periodic. We will show that $x$ is not chain recurrent.

Let $W_{1}$ denote the component of the complement (in $I$ ) of the set of periodic points of $f$ with $x \in W_{1}$. Let $W$ be the unique open interval such that $\bar{W}=\bar{W}_{1}$. Then either $x \in W$ or $x$ is an endpoint of $I$ and an endpoint of $W$.

By Lemma 1, to show that $x$ is not chain recurrent for $f$, we may show that $x$ is not chain recurrent for $f^{h}$. Hence, without loss of generality, we may assume that $W$ satisfies the hypothesis of Lemma 5 , and $f^{n}(y)<y$ for every $y \in W$ and every positive integer $n$.

Let $p$ denote the left endpoint of $W$. Then $f(p)=p$ and $x \neq p$.

Let $K=\cup_{n=0}^{\infty} f^{n}([p, x])$. Note that $K$ is an interval whose right endpoint is $x$ and $f(K) \subset K$. Let $a$ denote the left endpoint of $K$.

We claim that $x \notin \overline{f(K)}$. It follows from Lemma 5 and the definition of $K$ that $x \notin f(K)$. Thus, to prove the claim, it suffices to show that $f(a) \neq x$ (since $f(\bar{K})$ $=\overline{f(K)}$. If $a \in K$ it follows that $f(a) \neq x$, so we may assume that $a \notin K$. Note that $a \in \overline{f(K)}=f(\bar{K})$, but $a \notin f(K)$ (since $f(K) \subset K$ ). Thus, since $a$ is the only element of $\bar{K} \backslash K, f(a)=a$. Hence $f(a) \neq x$. This establishes our claim that $x \notin \overline{f(K)}$. 
We will show that $\overline{f(K)}$ can be extended to a positively chain invariant set which does not contain $x$ and does contain $f(x)$. Once this is shown. it follows from Lemma 3 that $x$ is not chain recurrent.

Note that $a$ (the left endpoint of $K$ ) is also the left endpoint of $f(K)$. Let $c$ denote the right endpoint of $f(K)$. Then $a<c<x$. Let $b$ denote the midpoint of the interval $[c, x]$. Then $f([a, b]) \subset[a, b]$.

If $f(a) \neq a$ or $a=0$ then by Lemma $4,[a, b]$ is positively chain invariant and, by Lemma 3, $x$ is not chain recurrent.

Thus we may assume that $f(a)=a$ and $a \neq 0$. Since $f$ and $f^{2}$ are uniformly continuous, there is a $\delta_{1}>0$ such that if $\left|v_{1}-y_{2}\right|<\delta_{1}$ then $\left|f\left(y_{1}\right)-f\left(y_{2}\right)\right|<b-a$ and $\left|f^{2}\left(y_{1}\right)-f^{2}\left(y_{2}\right)\right|<b-a$. Also, there is a $\delta_{2}>0$ with $\delta_{2}<\delta_{1}$ such that if $\left|y_{1}-y_{2}\right|<\delta_{2}$ then $\left|f\left(y_{1}\right)-f\left(y_{2}\right)\right|<\delta_{1}$.

Let $y \in\left(a-\delta_{2}, a\right)$. We will show that $f^{h}(y)<b$ for every positive integer $k$. Suppose that for some positive integer $k, f^{h}(y) \geqslant b$. Then for some nonnegative integer $r$ with $r<k, f^{r}(y) \in\left(a-\delta_{2}, a\right)$ and $f^{r+1}(r) \leqslant a-\delta_{2}$. Let $z=f^{r}(y)$. Then $f(z)<z$. Also. if $m=k-r$ then $f^{m}(z)>z$.

By Lemma 6, there is a point $s_{0} \in I$ such that $f^{\prime}(z)>s_{0}$ for $i=0.2,4 \ldots m-2$. and $f^{\prime}(z)<s_{0}$ for $i=1.3 .5 \ldots \ldots m-1$. Since $f(z)<s_{0}$ and $z \in\left(a-\delta_{2}, a\right)$. it follows from the choice of $\delta_{2}$ that $s_{0} \in\left(a-\delta_{1}, a\right)$.

Note that the points $z, f^{2}(z), f^{4}(z) \ldots \ldots f^{m} z(z)$ are all to the right of $s_{0}$. None of these points can be in the interval $[a, b]$ since $f([a, b]) \subset[a, b]$. Hence. it follows from the choice of $\delta_{1}$ (and by induction) that each of these points is in the interval $\left(s_{0}, a\right)$. Thus, by choice of $\delta_{1}, f^{\prime \prime \prime}(z)<b$. This is a contradiction since $f^{\prime \prime \prime}(z)=$ $f^{h}(y) \geqslant b$. Thus, for every $y \in\left(a-\delta_{2}, a\right)$ and every positive integer $k . f^{h}(l)<b$.

Let $K_{1}=\cup_{h=0}^{x} f^{h}\left(\left[a-\delta_{2}, a\right]\right)$. Here, in case $a-\delta_{2}<0$. we make the convention that $f^{h}(S)=f^{h}(S \cap I)$. Then $K_{1}$ is an interval and for all $y \in K_{1}, y \leqslant b$. Let $a_{1}$ denote the left endpoint of $K_{1}$. If $a_{1}=0$ or $f\left(a_{1}\right)>a_{1}$ then the interval $\left[a_{1}, b\right]$ satisfies the hypothesis of Lemma 4 and thus, $x$ is not chain recurrent. Hence, we may assume that $a_{1} \neq 0$ and $f\left(a_{1}\right)=a_{1}$.

Let $K_{2}=\cup_{h: 00}^{x} f^{h}\left(\left[a_{1}-\delta_{2}, a_{1}\right]\right)$. Note that if $y \in\left(a_{1}-\delta_{2}, a_{1}\right)$ then $f^{h}(y)<h$ for every positive integer $k$. This follows by the same proof used above to show that if $y \in\left(a-\delta_{2}, a\right)$ then $f^{h}(y)<b$ for every positive integer $k$. Thus, for all $y \in$ $K_{2}, y \leqslant b$. Let $a_{2}$ denote the left endpoint of $K_{2}$. If $a_{2}=0$ or $f\left(a_{2}\right)>a_{2}$ then the interval $\left[a_{2}, b\right]$ satisfies the hypothesis of Lemma 4 and $x$ is not chain recurrent. Hence, we say assume that $f\left(a_{2}\right)=a_{2}$.

We repeat the above argument inductively forming points $a_{n}$ with $a_{n+1} \leqslant a_{n}-\delta_{2}$. Since the interval $[0,1]$ has finite length, for some positive integer $n$ we must have either $a_{n}=0$ or $f\left(a_{n}\right)>a_{n}$. It follows that the interval $\left[a_{n}, b\right]$ satisfies the hypothesis of Lemma 4 and $x$ is not chain recurrent. Q.E.D.

\section{REFERENCES}

1. L. Block. Mappings of the intercal with fimitely many perioduc points have zero e'ntrops. Proc. Amer. Math. Soc. 67 (1977), 357-36().

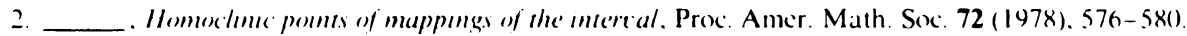

3. C. Conley. The grade'nt structure of a flow. I. IBM Rescarch. RC 3932 (\#17806). Yorktoun Heights, N. Y., July 17. 1972. 
4. Isolated invariant sets and the Morse index. CBMS Regional Conf. Ser. in Math.. no. 38. Amer. Math. Soc., Providence, R. I., 1976

5. E. M. Coven and (;. A. Hedlund. Continuous maps of the interval whose periodic points form a closed set. Proc. Amer. Math. Soc. 79 (1980), 127-133.

6. E. M. Coven and Z. Nitecki. Nonwandering sets of the pow'ers of maps of the interval. Ergodic Theory Dynamical Systems 1 (1981), 9-31.

7. T. Li, M. Misiurewicz, (j. Pianigiani and J. Yorke, Odd chaos, preprint.

8. Z. Nitecki. Maps of the interval nith closed periodic set. Proc. Amer. Math. Soc. 85 (1982), 451-456.

9. Topological dinamics on the interval. Ergodic Theory and Dynamical Systems II (College

Park. Md. 1979-1980). Progress in Math., vol. 21. Birkhauser, Boston. 1982, pp. 1-73.

10. Jin-Cheng Ziong. Continuous self-maps of the closed interval nhose periodic points form a closed set. J. China Univ. Sci. and Tech. 11 (1981), 14-23.

Department of Mathematics, University of Florida, Gainsville. Florida 32611

Department of Mathematics, North Carolina State University, Raleigh, North Carolina 27650 